\title{
Prevalencia de infección por VIH en la población adulta en México: una epidemia en ascenso y expansión
}

\author{
José Luis Valdespino, MC, MSP,(1) Ma. de Lourdes García-García, MC, D en C, (2) \\ Carlos J Conde-González, QBP, M en C, D en C, (2) Gustavo Olaiz-Fernández, MC, MSP, ${ }^{(3)}$ \\ Oswaldo Palma,Act, ${ }^{(2)}$ Jaime Sepúlveda, MC, M en C, D en C.(2)
}

\author{
Valdespino JL, García-García ML, Conde-González CJ, \\ Olaiz-Fernández G, Palma O, Sepúlveda J. \\ Prevalencia de infección por VIH en la población adulta \\ en México: una epidemia en ascenso y expansión. \\ Salud Publica Mex 2007;49 supl 3:S386-S394.
}

\section{Resumen}

Objetivo. ONUSIDA ha reconocido que calcular la magnitud de la infección porVlH en los planos nacional o regional es importante desde el punto de vista de la evaluación, planeación de programas y supervisión. Al adoptar esta recomendación, el propósito de este trabajo fue cuantificar la magnitud de la infección en la población general adulta mexicana a partir de los datos consignados en la ENSA 2000, la cual permitió determinar la prevalencia de infección por VIH en la población general y los principales factores relacionados. La disponibilidad de datos de la Encuesta Seroepidemiológica proveyó además la oportunidad de conocer las tendencias en el periodo entre las dos encuestas (1987-2000). Material y métodos. Se seleccionó de manera aleatoria a 21271 individuos de 20 años de edad o mayores, de ambos sexos, estudiados en la ENSA 2000 para lgG anti-VIHI/2 (ELISA), confirmados por inmunoelectrotransferencia, y los principales factores relacionados con seropositividad. Las muestras se procesaron en el Instituto Nacional de Salud Pública en 2005. Resultados. La prevalencia para anticuerpos anti-VIH-I fue de $0.25 \%$. Los principales factores vinculados con la infección fueron las edades más jóvenes, sexo masculino, vivir en el Distrito Federal, escolaridad de licenciatura o mayor y ser soltero, divorciado o separado. Conclusiones. La seroprevalencia en hombres adultos muestra un incremento de 10
Valdespino JL, García-García ML, Conde-González CJ, Olaiz-Fernández G, Palma O, Sepúlveda J.

Prevalence of HIV infection among adult population in Mexico: an increasingly expanding epidemic. Salud Publica Mex 2007;49 suppl 3:S386-S394.

\begin{abstract}
Objective. UNAIDS has recognized that estimating HIV infection at a national or regional level is important for evaluation, program planning and advocacy. Following this recommendation, the purpose of this study was to estimate the magnitude of HIV infection prevalence among adults from the general population using information from the National Health Survey (ENSA 2000), a probabilistic, multistage, stratified, cluster household survey conducted by the Mexican Ministry of Health between November 1999 and June 2000.Availability of information from the Seroepidemiological Survey conducted in 1987 allowed for the estimation of trends between the two surveys (1987-2000). Material and Methods. A total of $2127 \mathrm{I}$ males and females 20 years or older included in the ENSA 2000 were randomly selected and studied for anti-HIV I/2 lgG (ELISA), confirmed by immunoelectrotransference (WB), and for risk factors associated with HIV infection. Samples were processed in the National Institute of Public Health in 2005. Results. Prevalence for HIV infection was $0.25 \%$. The main factors associated with infection were younger ages, male gender, residence in the Federal District, college education or higher, and being single or divorced. Conclusions. Seroprevalence among adults shows a ten-fold increase as compared with the results of the national seroprevalence survey of 1987. Although the
\end{abstract}

(I) Instituto Nacional de Salud Pública; actualmente en el Laboratorio de Biológicos y Reactivos de México (Birmex).

(2) Instituto Nacional de Salud Pública. México.

(3) Secretaría de Salud. México.

Fecha de recibido: 31 de marzo de 2006 - Fecha de aceptado: 16 de mayo de 2007

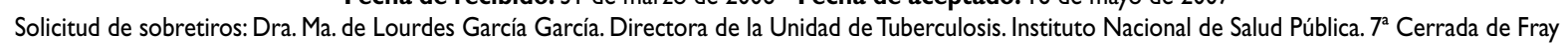
Pedro de Gante No. 50; Col. Sección XVI, Delegación Tlalpan, I 4000 México, DF, México.

Correo electrónico: garcigarml@gmail.com 
veces en comparación con la encuesta nacional de 1987. Si bien la epidemia continúa concentrada en hombres que tienen relaciones sexuales con hombres, existe evidencia de diseminación a la población heterosexual.

Palabras clave: VIH/SIDA; estudios seroepidemiológicos; población; encuesta; México epidemic continues to be concentrated among men who have sex with men, results provide evidence of dissemination towards the heterosexual population.

Key words:HIVIAIDS; seroepidemiologic studies; population; survey; Mexico
L os esfuerzos mundiales aún son insuficientes para contener la epidemia de SIDA en el mundo. De acuerdo con los datos de ONUSIDA, en diciembre de 2005 vivían en el mundo 40.3 (36.7-45.3) millones de personas infectadas por VIH, de los cuales 17.5 millones (16.2-19.3) eran mujeres y 2.3 millones (2.1-2.8) niños. Este número de personas afectadas duplica el informado en 1995 (19.9 millones). Casi 5 millones de infecciones ocurrieron en 2005, 4.2 millones (3.6-5.8) en adultos. La prevalencia notificada de infección por VIH global fue de $1.1 \%$ (1.0-1.3) y la más alta se registró en África subsahariana $(7.2 \%, 6.6-8.0)$. En América Latina se habían acumulado 1.8 millones (1.4-2.4) de infecciones hasta ese mismo año, 200000 (130 000-360 000) tan sólo en 2005. En esta región se calculó la prevalencia de infección en $0.6 \%(0.5-0.8){ }^{1}$

En México, hasta el 15 de noviembre de 2005, se habían notificado 98933 casos de SIDA y 180000 personas vivían con $\mathrm{VIH} .{ }^{2}$ Se considera que la epidemia en México se halla concentrada en grupos que mantienen prácticas de riesgo. Casi $90 \%$ de los casos de SIDA notificados en registros oficiales se han atribuido a las relaciones sexuales sin protección y alrededor de la mitad de ellas a las relaciones sexuales entre varones. ${ }^{2}$ Sin embargo, la transmisión heterosexual representa $44.5 \%$ de los casos notificados y el número creciente de pacientes mujeres (62 de los 987 individuos [5.9\%] en $1987^{3}$ a 837 de los 3240 [20\%] en 2004, ${ }^{4}$ esto es, de una relación hombremujer de 16:1 en 1987 a una de 4:1 en 2004) subraya la importancia de este grupo poblacional. La transmisión por transfusión sanguínea, que representaba hasta $17 \%$ de los casos en la década de los años ochenta, ${ }^{5}$ ocasiona en la actualidad 3.6\% de los casos. La transmisión en consumidores de drogas intravenosas es relativamente menor $(0.7 \%)$. Sin embargo, el papel que juega esta vía de transmisión, en particular en la frontera con Estados Unidos, es cada vez más importante. ${ }^{6}$

En México se efectúo en 1986 la primera Encuesta Nacional de Salud (ENSA-I) como antecedente del establecimiento de un Sistema Nacional de Encuestas de Salud en el que se incluyen diversas encuestas con objetivos específicos y complementarios. Después de la ENSA-I se realizó en 1987 la Encuesta Nacional
Seroepidemiológica, que estudió por primera ocasión en México la prevalencia de infección por VIH en la población general. En ese año se recolectaron 78536 sueros mediante un marco muestral probabilístico de hogares. Para la determinación de anticuerpos contra VIH-1 se seleccionaron 10921 sueros de hombres mayores de 15 años y se detectaron cuatro positivos, con una seroprevalencia en varones adultos para 1987 de $0.04 \% .^{7}$

En Estados Unidos, la NHANES III examinó a 11203 individuos de 18 a 59 años de edad entre 1988 y 1994. La prevalencia general fue de $0.32 \%$ (IC95\%: 0.20-0.51), pero se identificó que la prevalencia en estadounidenses de origen mexicano era de $0.37 \%$ (IC95\%:0.18-0.75), más alta que la general aunque menor que la encontrada en la población negra $\left(1.10 \%\right.$, IC95\%: 0.71-1.71). ${ }^{8}$

ONUSIDA ha reconocido que cuantificar la magnitud de la infección por VIH a escala nacional o regional es importante en términos de la evaluación, planeación de programas y supervisión. ${ }^{9}$ En concordancia con esta recomendación, este trabajo determina las dimensiones de la infección en la población general adulta mexicana con base en los datos que suministra la ENSA 2000, la cual permitió precisar la prevalencia de infección por VIH en la población general y los principales factores adjuntos. La disponibilidad de datos de la Encuesta Seroepidemiológica proveyó además la oportunidad de conocer las tendencias observadas en el periodo entre las dos encuestas (1987-2000).

\section{Material y métodos}

\section{Diseño}

La Encuesta Nacional de Salud 2000 (ENSA 2000), cuyo diseño se ha descrito con anterioridad, ${ }^{10}$ estuvo a cargo de la Secretaría de Salud y se efectuó entre noviembre de 1999 y junio de 2000. De forma sinóptica, incluyó 43479 hogares (con un total de 90916 individuos) seleccionados al azar por probabilidad multietápica con el fin de ser representativa de la población mexicana civil, no institucionalizada, en los planos nacional y estatal. La recolección de la información se le encomendó a perso- 
nal entrenado, quien investigó información sociodemográfica, del estado actual de salud, uso de servicios de salud y comportamiento de los participantes, y recogió además muestras biológicas. Para investigar el consumo de drogas se planteó la siguiente pregunta: "Me podría decir si ha tomado, usado o probado por indicación médica o por alguna otra razón." Las respuestas fueron “opiáceos; tranquilizantes para los nervios; sedantes o barbitúricos para dormir; anfetaminas o estimulantes para adelgazar o dar energía; marihuana; cocaína; heroína; alucinógenos como hongos, peyote, mezcalina, LSD; inhalables como thiner, pegamento, pintura o gasolina". Para cada una de las respuestas se ofrecieron las opciones "sí, no, o no responde". No hubo más preguntas acerca del consumo de drogas. En el caso del uso del condón, se formuló para hombres y mujeres de 20 a 49 años de edad la pregunta: “¿Qué están actualmente haciendo usted o su pareja para no tener hijos?" Las respuestas, con la aclaración de poder anotar más de una opción, fueron las siguientes: "operación femenina o ligadura; operación masculina o vasectomía; pastillas o píldoras; inyecciones; norplant; DIU; preservativo o condón; óvulos, jaleas o espumas; ritmo, calendario, abstinencia periódica, termómetro, Billings; retiro o coito interrumpido; otro; no responde". No se hicieron más preguntas sobre el uso del condón. El presente estudio se basa en la información de 21271 individuos de 20 años de edad o mayores, de ambos sexos, seleccionados de manera aleatoria.

\section{Obtención, transporte y conservación de muestras biológicas}

Las muestras se colectaron en los domicilios respectivos. La sangre se obtuvo mediante venopunción al vacío con tubos Vacutainer SST (Becton-Dickinson), que se centrifugaron en los tubos. Con posterioridad se almacenaron en refrigeración a $4^{\circ} \mathrm{C}$ por no más de cinco días y se trasladaron al banco de sueros del INSP en hieleras con refrigerantes para la separación de los sueros. Las muestras se conservaron hasta su procesamiento en cámaras de congelación con vapores de nitrógeno líquido a $-150^{\circ} \mathrm{C}$

\section{Pruebas diagnósticas de laboratorio}

Las muestras se procesaron mediante la técnica $\operatorname{IgG}$ anti-VIH 1/2 (Axsym, Abbott Laboratories, EUA). Las muestras repetidamente reactivas se procesaron mediante la prueba Western blot (Pasteur Diagnostics, Francia) y se interpretaron según los criterios que recomienda la ASTPHLD/CDC. ${ }^{11}$

\section{Análisis}

Los datos se ajustaron para considerar las probabilidades diferenciales de selección y no respuesta. Para el análisis estadístico se calculó la seroprevalencia de anticuerpos anti-VIH, de acuerdo con algunas características demográficas, socioeconómicas y geográficas. Para conocer la relación entre algunos factores concomitantes y la seropositividad a infección por VIH se calcularon las razones de momios (RM) y los intervalos de confianza al 95\%. En los procedimientos estadísticos se cuantificaron los factores de expansión poblacional. Los datos se procesaron con el paquete estadístico SAS 9.1 (SAS Institute, Cary NC, EUA) y SUDAAN 7.5.6. Las razones de momios y los intervalos de confianza se obtuvieron con este último paquete estadístico, que considera el tipo de muestreo utilizado en el diseño muestral de esta encuesta.

\section{Aspectos éticos}

El protocolo obtuvo la aprobación de las comisiones de investigación, ética y bioseguridad del Instituto Nacional de Salud Pública. De modo adicional, la recolección y presentación de los datos se efectuaron bajo la observancia de los principios de confidencialidad y reserva que señala la Ley de Información Estadística y Geográfica. ${ }^{12}$

\section{Resultados}

De los 45294 sujetos de 20 años o mayores con muestra de suero de la ENSA 2000 se seleccionaron por muestreo aleatorio simple 21271 sueros para estudio de anticuerpos contra VIH-1. La seroprevalencia de anticuerpos HIV-1 ya ponderada en la población mexicana adulta (mayor de 20 años) es de $0.25 \%$ (intervalo de confianza al 95\%: 0.14-0.45). Este porcentaje corresponde a 130138 adultos mexicanos infectados por este agente (IC95\%: 72 998-231 889).

La prevalencia de infección varía de acuerdo con la edad y es mayor en los sujetos más jóvenes. El grupo de 20 a 29 años tiene la seroprevalencia más elevada: $0.32 \%$ (IC95\%: 0.14-0.73). La frecuencia decrece conforme a la edad y en el grupo de 50 años o más es de 0.07 (IC95\%: 0.03-0.2) (figura 1, panel A).

La prevalencia es mayor en los hombres $(0.48 \%$, IC95\%: $0.26-0.92)$ que en las mujeres $(0.05 \%, 0.02-0.13)$. La mayor prevalencia en hombres que en mujeres se observa en todos los grupos de edad, si bien a edades mayores la diferencia entre ambos sexos es menor. En los hombres son los más jóvenes, los de 20 a 29 años, 
(A) Prevalencia de anticuerpos contra VIH por grupo de edad e intervalos de confianza

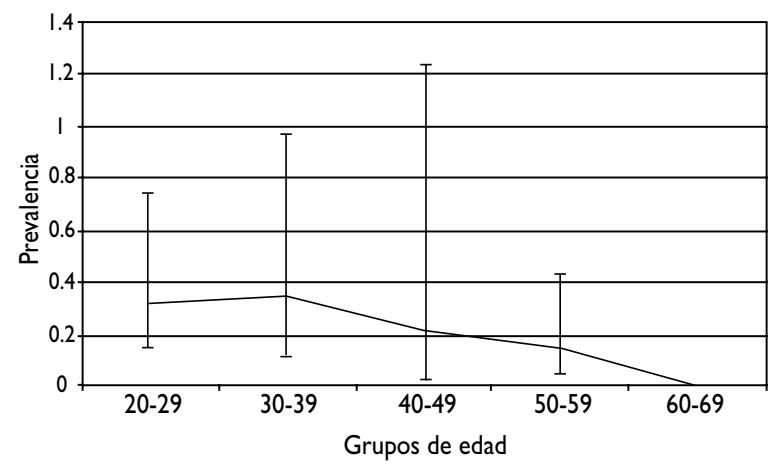

(B) Prevalencia de anticuerpos contra VIH-I por grupos de edad y sexo.

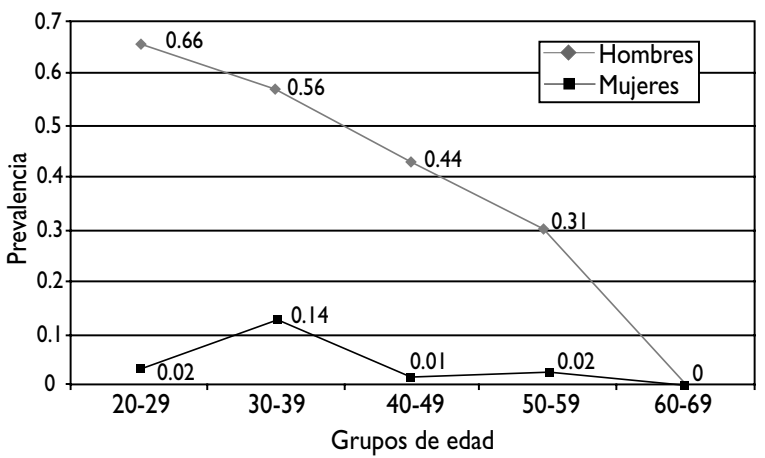

Figura I. Prevalencia de anticuerpos contra ViH-I en INDIVIDUOS MAYORES DE 20 aÑos. MÉXICO, ENSA 2000

quienes muestran la prevalencia más alta $(0.66 \%$, IC95\%: 0.28-1.57). En cambio, en las mujeres la prevalencia más alta se identifica en edades un poco mayores, de 30 a 39 años (0.14\%, IC95\%: 0.04-0.50) (figura 1, panel B).

Mediante análisis crudo, los principales factores vinculados con la infección fueron edad menor a 50 años (RM=4.07, IC95\%: 1.25-13.2), sexo masculino (RM=10.15, IC95\%: 3.16-32.6), vivir en el Distrito Fede$\operatorname{ral}(\mathrm{RM}=4.54$, IC95\%: 0.95-21.6), tener licenciatura o un nivel de escolaridad mayor (RM=25.6, IC95\%: 2.5-259), estar separado, divorciado, o soltero, ser económicamente activo (RM=5.6, IC95\%: 1.46-21.8), no tener pareja (RM=21.7, IC95\%: 5.79-81.2) y referir consumo de heroína ( $\mathrm{RM}=9.03$, IC95\%: 0.94-86.4) (cuadro I). Los 20 casos que refirieron haber tomado, consumido o probado heroína correspondieron a siete individuos de la zona norte, seis de la zona centro, cinco de la zona sur y dos del Distrito Federal.

$\mathrm{Al}$ ajustar por edad persistió el mayor riesgo en hombres (RM=10.14, IC95\%: 3.15-32.6). Al ajustar por edad y sexo se demostró un menor riesgo en los individuos de más edad (para los mayores de 50 años, $\mathrm{RM}=0.23$, IC95\% 0.06-0.86) y mayor riesgo entre los que tuvieron mayor nivel de instrucción (para licenciatura o posgrado, $\mathrm{RM}=12.8$, IC95\%: $1.01-160.8)$ y para los separados, divorciados y solteros ( $\mathrm{RM}=10.6,17.3$ y 15.3 , respectivamente).

En los hombres, las características que se vincularon con seropositividad fueron ser soltero ( $\mathrm{RM}=16.4$, IC95\%: 7.2-90.4), divorciado (RM=37.3, IC95\%: 6.8-245.4), separado ( $\mathrm{RM}=23.6$, IC95\%: 2.5-185) y no tener pareja (RM=16.5, IC95\%: 4.1-65.8) (cuadro II).

\section{Discusión}

Los resultados de este estudio permiten calcular la prevalencia de infección por VIH-1 en la población general, la cual resultó de $0.25 \%$ (IC95\%: 0.14-0.45). La prevalencia informada en esta encuesta se halla por debajo de la que previó la ONUSIDA para la región $(0.6 \%$, IC95\%: $0.5-0.8)^{1}$ y por debajo de los resultados informados en la tercera Encuesta de Salud y Nutrición de Estados Unidos (NHANES III) para la población estadounidense de origen mexicano: 0.37\% (IC95\%: 0.18-0.75; 0.54\% [IC95\%: 0.27-1.09] en hombres y 0.17\% [IC95\%: 0.05-0.57] en mujeres), aunque en ella los intervalos de confianza comprenden los resultados informados en este estudio. ${ }^{8}$

Si bien la seroprevalencia es menor que en otras regiones, como el sureste asiático o África, la comparación con la Encuesta Seroepidemiológica de 1987 revela un incremento de casi 10 veces entre ambas encuestas. En esta última, la seroprevalencia en hombres mayores de 15 años fue de $0.04 \% 7$ y en la presente encuesta la seroprevalencia en hombres mayores de 20 años fue de $0.48 \%$. Por lo tanto, los resultados indican que la epidemia de VIH en hombres de la población general en México muestran una tendencia ascendente.

Estos datos son consistentes con estudios realizados en donadores y mujeres embarazadas; tales protocolos, si bien tienen limitaciones (estudiar a personas que en general son más jóvenes y sanas que la población en general) no padecidas en las encuestas probabilísticas, son útiles como indicadores de la frecuencia de infección en la población general. Los resultados en donadores de sangre informados hasta 1994 se referían a 1.5 millones de determinaciones, con prevalencia de infección confirmada mediante Western blot de $0.05 \%$. La tendencia de seroprevalencia había 


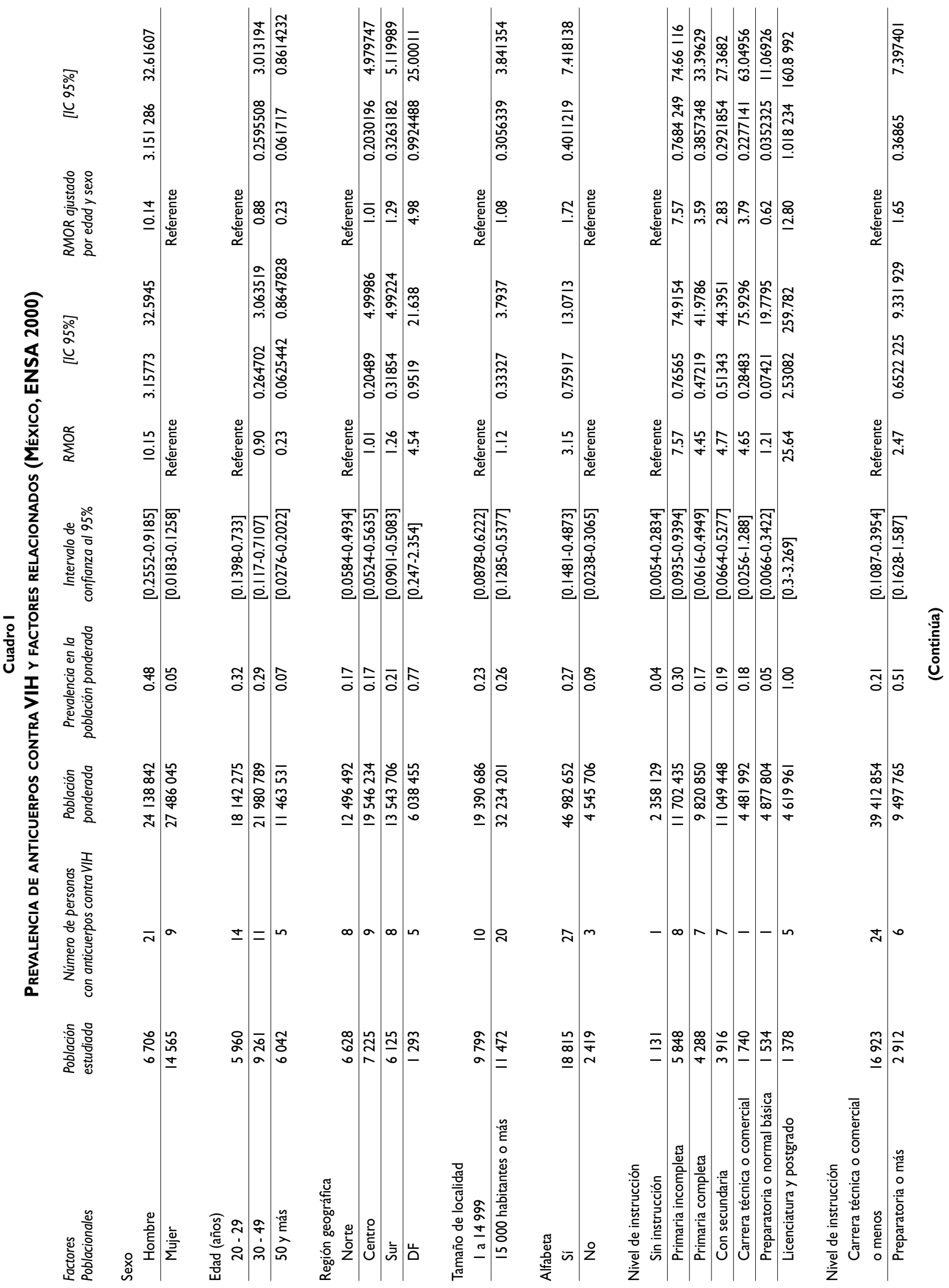



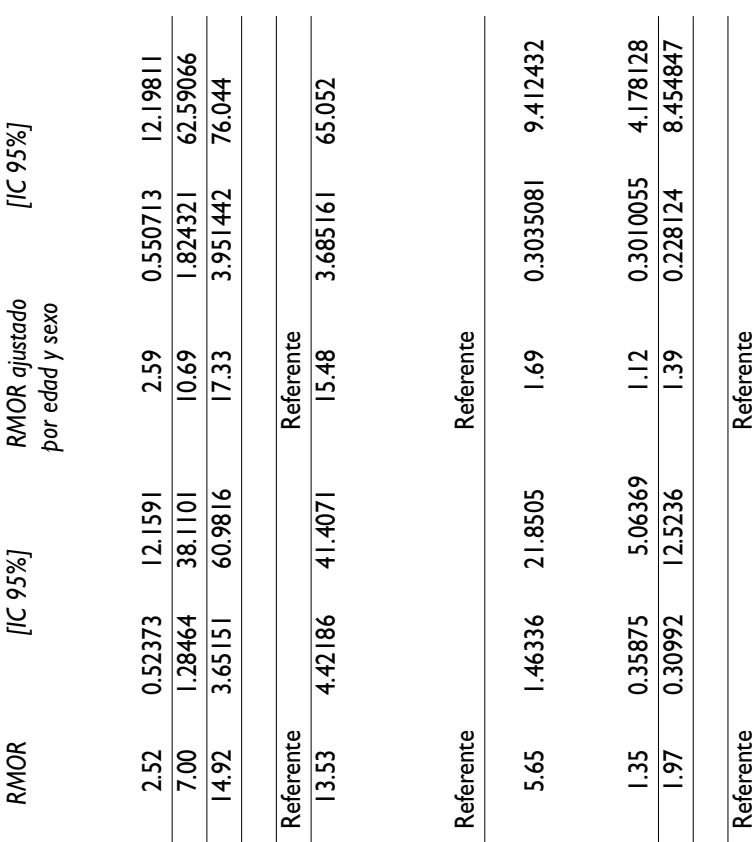

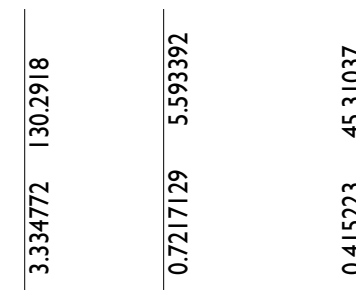

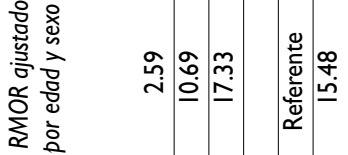

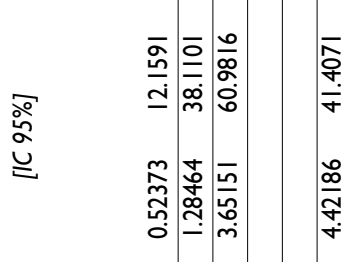

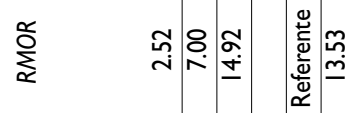

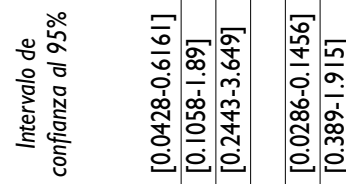

ฮิْ

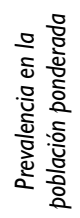

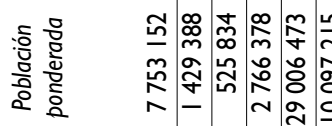

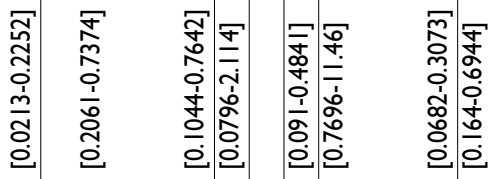

응

잉

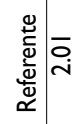

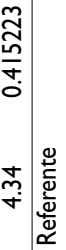

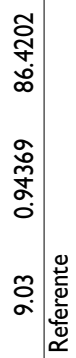

列

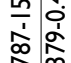

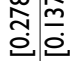

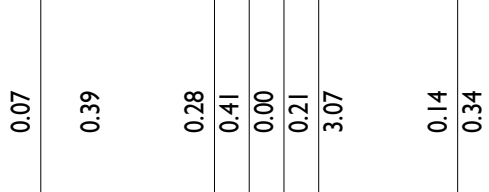

অ

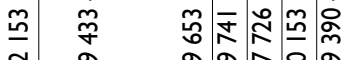

ส ส

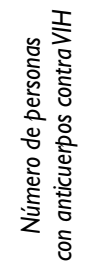

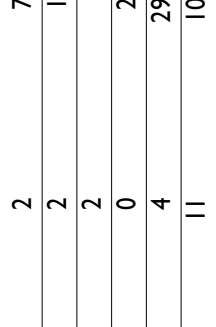

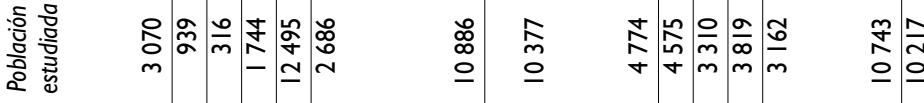

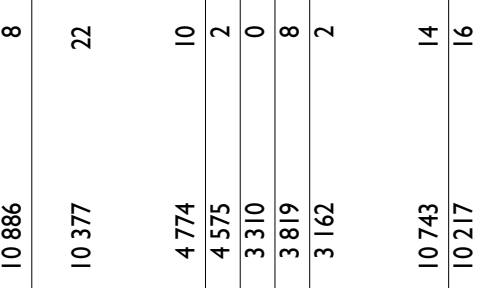

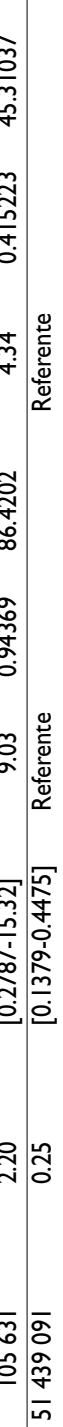

文 


\section{Cuadro II}

Prevalencia de anticuerpos contra ViH y factores de riesgo en hombres (México, ENSA 2000)

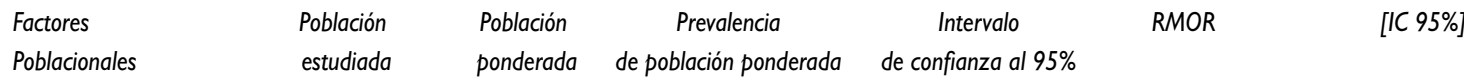

Estado civil (hombres)

\begin{tabular}{lrrrrrrr} 
Vive con su pareja & 964 & 3608340 & 0.33 & {$[0.0785-1.346]$} & 4.93 & 0.8951621 & 32.52662 \\
\hline Separado & 146 & 386836 & 1.30 & {$[0.2124-7.506]$} & 23.63 & 2.534834 & 185.063 \\
\hline Divorciado & 74 & 207005 & 2.42 & {$[0.6026-9.233]$} & 37.28 & 6.824261 & 245.4282 \\
\hline Viudo & 288 & 530903 & 0.00 & & & & \\
\hline Casado & 4215 & 13686797 & 0.06 & {$[0.0207-0.1774]$} & Referente & & 7.21172 \\
\hline Soltero & 1012 & 5692729 & 1.53 & {$[0.6728-3.425]$} & 16.42 & 90.45201 \\
\hline Total & 6699 & 24112610 & 0.49 & {$[0.2555-0.9195]$} & &
\end{tabular}

Edad de la primera relación sexual (para hombres de 20 años o más)

\begin{tabular}{lrrrrrrr}
$1 \mid-15$ & $128 \mid$ & 4308347 & 0.88 & {$[0.2884-2.652]$} & 2.02 & $0.397072 \mid$ & $10.23 \mid 85$ \\
\hline $16-20$ & 3618 & 12999517 & 0.38 & {$[0.1412-1.039]$} & 0.87 & 0.1747518 & 4.379102 \\
\hline $21-30$ & 1185 & 4322367 & 0.68 & {$[0.1944-2.32]$} & Referente & & \\
\hline 30 y más & 68 & 144944 & 0.00 & & &
\end{tabular}

Anticoncepción (para hombres de 20 a 49 años de edad)

\begin{tabular}{lrrrcrcr} 
Sí & 2045 & 7886937 & 0.15 & {$[0.0564-0.4157]$} & Referente & \\
\hline No & 1532 & 5978765 & 0.33 & {$[0.1053-1.053]$} & 2.18 & 0.6142369 & 7.749668 \\
\hline No tiene pareja & 569 & 2872191 & 2.60 & {$[1.056-6.243]$} & 17.36 & 4.349452 & 69.30234 \\
\hline No responde & 113 & 500575 & $0.5 \mathrm{I}$ & {$[0.0691-3.597]$} & 3.31 & 0.3606756 & $30.3351 \mathrm{I}$
\end{tabular}

\begin{tabular}{|c|c|c|c|c|c|c|c|}
\hline \multicolumn{8}{|c|}{ Métodos anticonceptivos (para hombres de 20 a 49 años de edad) } \\
\hline Preservativo o condón & 263 & I I 172549 & 0.04 & {$[0.0059-0.3067]$} & Referente & & \\
\hline \multicolumn{8}{|l|}{ Otro (operación masculina } \\
\hline \multicolumn{8}{|l|}{ o vasectomía , ligadura, } \\
\hline \multicolumn{8}{|c|}{ pastillas, inyecciones, norplant, } \\
\hline \multicolumn{8}{|c|}{ DIU, u óvulos, jaleas, espumas, } \\
\hline \multicolumn{8}{|c|}{ ritmo, retiro o coito } \\
\hline interrumpido, etc.) & 1782 & 6714388 & 0.17 & {$[0.0608-0.4891]$} & 4.07 & 0.4257103 & 38.90898 \\
\hline
\end{tabular}

permanecido estable con fluctuaciones de 0.05 a $0.09 \%$. En fecha más reciente se ha observado un incremento de la prevalencia en donadores voluntarios. En información que proporciona la Secretaría de Salud a la Organización Panamericana de la Salud sobre más de un millón de unidades estudiadas anualmente entre 2000 y 2003, la prevalencia de infección por VIH fluctuó entre 0.28 y $0.36 \%{ }^{13}$ y en un informe sobre la seguridad de las reservas sanguíneas durante los años 1999 a 2003, en que se analizaron más de 5 millones de unidades, la prevalencia de infección por VIH osciló entre 0.24 y $0.30 \% .{ }^{14}$ Las encuestas centinela de VIH en mujeres embarazadas se iniciaron en 1988 y se procesaron 3284 sueros en 15 ciudades entre 1994 y 1995, de los cuales sólo dos resultaron positivos (seroprevalencia de $0.06 \%) .{ }^{7}$ Con posterioridad a esta fecha son escasos los estudios publicados, aunque se prevé que la prevalencia en mujeres ha pasado de $0.04 \%$ en 1990 a $0.09 \%$ en 1997. ${ }^{15}$ En un estudio de 310 mujeres que acudieron a control prenatal en Yucatán no se identificó a ninguna infectada, ${ }^{16}$ en tanto que en un hospital de referencia en la Ciudad de México en el periodo de 1994 a 1996 se reconoció a 19 mujeres infectadas. ${ }^{17}$

Algunas de las características relacionadas con la infección y reconocidas en este estudio (ser hombre, joven, soltero, divorciado o separado y tener residencia habitual en el Distrito Federal) coinciden con las vinculadas con seropositividad en hombres adultos que acudieron a un centro de detección de anticuerpos en la Ciudad de México entre 1988 y 1989 y en un estudio llevado a cabo en seis ciudades (Acapulco, Guadalajara, Mérida, Ciudad de México, Monterrey y Tijuana) en 
lugares de reunión de hombres homosexuales en 1988. ${ }^{18}$ Estos factores perfilan el carácter de concentración de la epidemia en grupos específicos.

Destaca el hallazgo de un nexo con el consumo de heroína por el incremento del uso de drogas intravenosas que se ha descrito en años recientes para México, ${ }^{19}$ aunque debe señalarse que la relación sólo se observó en el análisis crudo y no en el análisis ajustado por edad y sexo. El problema de infección por VIH lo habían ya detectado otros autores, quienes publicaron frecuencias de infección en consumidores de drogas intravenosas de 0.6 a $4.01 \% .20-23$

La principal limitante de las encuestas basadas en hogares es la atribuida a las tasas de no respuesta. Ésta no parece ser una limitante en el caso de la ENSA 2000, ya que el análisis de la población mayor de 20 años de edad mostró distribución semejante a la de la población adulta mexicana que proporcionó el Censo de Población y Vivienda de 2000, ${ }^{24}$ por lo cual puede presuponerse que la tasa de no respuesta no afectó la representatividad nacional de los datos. Otro punto importante es que la ENSA 2000 excluye a la población institucionalizada, como los prisioneros, entre quienes el problema de infección por VIH se ha considerado preocupante; en realidad, se han notificado en este grupo frecuencias de infección de 0.01 a 1.2\%. ${ }^{21,25-27}$ Otro grupo excluido de las encuestas de hogares es el de las personas en situación de calle, en quienes también se han reconocido tasas elevadas de infección. ${ }^{28,29}$ La interpretación de los datos de esta encuesta debe considerar las características inherentes a los estudios de seroprevalencia. La prevalencia de infección mide las infecciones antiguas y las recientes y depende de la aparición de infecciones nuevas y la sobrevida de los enfermos. Un factor que podría contribuir al incremento de la prevalencia de infección es el de una mayor sobrevida en los sujetos que reciben antirretrovirales. La mayor sobrevida de estos pacientes puede determinar un número mayor de individuos infectados y no necesariamente un número mayor de infecciones nuevas. Existen datos preliminares de que la disponibilidad de fármacos antirretrovirales en México pudo reducir la mortalidad consecutiva al SIDA (entre los hombres de 25 a 34 años pasó de 20.5 por 100000 en 1996 a 16.7 por 100000 en $1999^{30}$ ) y aumentar la sobrevida de estos pacientes en cuando menos una unidad hospitalaria. ${ }^{31} \mathrm{El}$ análisis de las tendencias de casos y defunciones por SIDA muestra una disminución de los casos, mientras que las defunciones han tendido a estabilizarse, al tiempo que se observa un incremento de la prevalencia de infección por VIH (Figura 2). ${ }^{32,33}$ Por lo tanto, la estabilización de la mortalidad por SIDA podría explicar en parte el incremento de la prevalencia de infección por VIH entre ambas encuestas, aunque no explica el fenómeno en su totalidad. Por último, debe tomarse en consideración que se detectó sólo a 30 individuos infectados, por lo que la interpretación debe mesurarse en grado considerable, ya que el número limitado de observaciones conduce a que las comparaciones sean inestables desde el punto de vista estadístico. Esta limitante se confrontó en este estudio mediante el cálculo de intervalos de confianza para estimar los límites inferiores y superiores entre los cuales podrían encontrarse los parámetros poblacionales.

La mayor utilidad de este estudio se traduce en recomendaciones para la prevención de la diseminación de la infección. Es evidente que aún es prioritaria la institución de medidas que incidan en la modificación de los estilos de vida, en especial los referentes al uso del condón y el consumo de drogas intravenosas. En México, si bien las medidas orientadas a promover prácticas de sexo seguro se iniciaron casi de manera simultánea con el diagnóstico del primer caso, su cobertura y por tanto su eficacia poblacional han sido limitadas, como lo demuestran diversos estudios en trabajadores migrantes, ${ }^{34}$ estudiantes, ${ }^{35}$ adolescentes, ${ }^{36}$ trabajadoras sexuales, ${ }^{37} \mathrm{y}$ hombres seleccionados de la población general. ${ }^{38}$ En una revisión reciente de estudios sobre el uso del condón se halló que si bien se ha incrementado su empleo, aún existe un alto porcentaje de relaciones sexuales no protegidas, en particular entre jóvenes. ${ }^{39}$ La inversión en prevención es todavía limitada, según lo demuestra un análisis de gastos a nivel nacional. ${ }^{40}$ En consecuencia, es prioritario realizar intervenciones integradas que incluyan de forma simultánea y enérgica la prevención, el tratamiento, la atención clínica y la mitigación del daño.

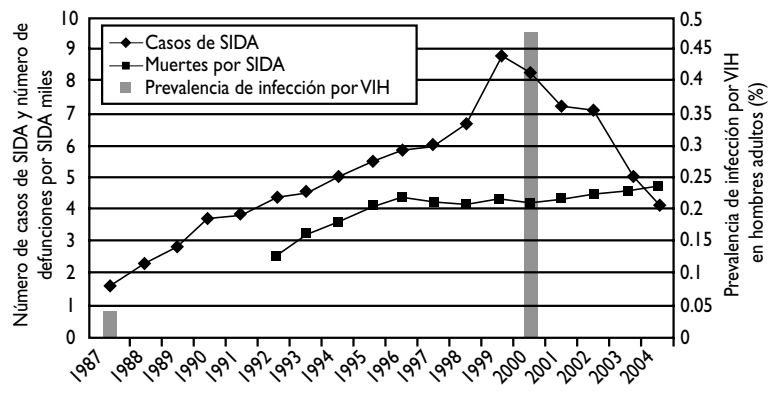

Fuentes: casos de SIDA (ref. 4); muertes por SIDA (ref. 39, 40); prevalencia de infección porVIH (1987: ref. 8, 2000: ENSA 2000).

Figura 2. Número de casos anuales de SiDA, númeRO DE DEFUNCIONES ANUALES POR SIDA Y PREVALENCIA De INFECCIÓN POR VIH en hombres adultos (México, | 987-2004) 


\section{Agradecimientos}

Este proyecto fue parcialmente financiado por CONACyT: 37212-M y MO244-9911. Los autores expresan su agradecimiento al personal de laboratorio TLC Santa García Cisneros, Biól. María Olamendi Portugal, Ing. Cruz Portugal García y Biól. Manuel Velázquez Meza por su valiosa labor.

\section{Referencias}

I.UNAIDS.AIDS Epidemic Update. December 2005. Ginebra: UNAIDS, 2005. 2. Centro Nacional para la Prevención y el Control delVIH/SIDA. El SIDA en cifras. México: CENSIDA, 2005.

3. Sector Salud. Situación del SIDA en Mexico. Datos actualizados hasta el I de diciembre de 1987. Dirección General de Epidemiología. Boletín Mensual de SIDA 1987;I(I0):192-200.

4. Consejo Nacional para la Prevención y Control delVIH/SIDA. Panorama Epidemiológico del VIH/SIDA e ITS en México. 3I de diciembre de 2004.

DF, México: CENSIDA, 2004.

5. Sepulveda-Amor J, del Río-Zolezzi A,Valdespino-Gómez JL, García-García M L,Velázquez-Velázquez L,Volkow P. The strategy for preventing HIVIAIDS transmission via the blood and its derivatives in Mexico. Salud Publica Mex 1995;37(6):624-35.

6. Magis-Rodríguez $\mathrm{C}$. Estudio sobre practicas de riesgo de infección por $\mathrm{VIH} / \mathrm{SIDA}$ en inyectores de drogas de la Cd. de Tijuana, BC. Rev Salud Front 1997;2:31-14.

7.Valdespino-Gómez JL, García-García M L, del Río-Zolezzi A, Loo-Méndez E, Magis-Rodríguez C, Salcedo-Álvarez RA. The epidemiology of AIDS/HIV in Mexico: from 1983 to March 1995. Salud Publica Mex 1995;37(6):556-7I. 8. McQuillan GM, Khare M, Karon JM, Schable CA,Vlahov D. Update on the seroepidemiology of human immunodeficiency virus in the United States household population: NHANES III, 1988- 1994. J Acquir Immune Defic Syndr Hum Retrovirol 1997;14(4):355-60.

9. UNAIDS/WHO.Working Group on Global HIVIAIDS and STI surveillance. Guidelines for measuring national HIV prevalence in population-based surveys. Ginebra: UNAIDS/WHO, 2005.

I0.Valdespino JL, Olaiz G, Mendoza L, Palma O,Velazquez O, et al. Encuesta Nacional de Salud 2000. Tomo I.Vivienda, población y utilización de servicios de salud. Cuernavaca, México: Instituto Nacional de Salud Pública, 2003.

II. Interpretation and use of the western blot assay for serodiagnosis of human immunodeficiency virus type I infections. MMWR Morb Mortal Wkly Rep 1989;38(Suppl 7): I-7.

12. Ley de Información Estadística y Geográfica. Diario Oficial de la Federación, 30 de diciembre, 1980.

13. Ramiro-Cruz J. Medicina transfusional en los países del Caribe y Lationamérica, 2000-2003. (THS/EV-2005/005-E).Washington,DC: Organización Panamericana de la Salud; 2005.

14.Vázquez-Flores J,Valiente-Banuet L, Marín L, Sánchez-Guerrero A. La seguridad de las reservas sanguíneas en la República Mexicana durante los años 1999 a 2003. Rev Inves Clin 2006;58(2): 101-108.

15. Guerra-Márquez A. EIVIH/SIDA y la mujer.Atención familiar (Órgano informativo del Departamento de Medicina Familiar) 2002;9(3).

16.Vera-Gamboa L, Gongora-Biachi R, Pavia-Ruiz N, Gaber-Osorno J, Lara-Perera D,Alonzo-Salomon L. Aceptabilidad para la detección de anticuerpos contra elVIH en un grupo de mujeres embarazadas de Yucatán, México. Ginecol Obstet Mex 2005;73(7):355-359.

17.Arredondo-García J, Hernández-Trejo M, Figueroa-Damián R, Ortiz-lbarra F,Ahued-Ahued J.SIDA y embarazo. Ginecol Obstet Mex 1997;66:8-12. 18. Izazola-Licea JA, Ávila-Figueroa RC, Gortmaker SL, del Río-Chiriboga C.
The homosexual transmission of HIV/AIDS in Mexico. Salud Publica Mex 1995;37(6):602-14.

19. Medina-Mora ME, Cravioto P,Villatoro J, Fleiz C, Galvan-Castillo F, TapiaConyer R. Drugs use among adolescents: results from the National Survey on Addictions, 1998. Salud Publica Mex 2003;45 (Suppl I):SI6-25.

20. Magis-Rodríguez C, Ruiz-Badillo A, Ortiz-Mondragón R, Loya-Sepúlveda M, Bravo-Portela M, Lozada-Romero R. Estudio sobre prácticas de riesgo de infección para VIH/SIDA en inyectores de drogas de la ciudad de Tijuana B.C. Biblioteca Virtual en Salud México. DF: México: ONUSIDA, 1997.

21. Guerena-Burgueno F, Benenson AS, Sepulveda-Amor J. HIV-I prevalence in selected Tijuana sub-populations. Am J Public Health 1991;81(5):623-5. 22. Magis-Rodríguez C, Brouwer KC, Morales S, Gayet C, Lozada R, OrtizMondragon R, et al. HIV prevalence and correlates of receptive needle sharing among injection drug users in the Mexican-U.S. border city of Tijuana.J Psychoactive Drugs 2005;37(3):333-9.

23. Rodríguez CM, Marques LF, Touze G. HIV and injection drug use in Latin America.Aids 2002;16 Suppl 3:S34-4I.

24. Olaiz G, Rojas R, Barquera S, Shamah T,Aguilar C, Cravioto P, et al. Encuesta Nacional de Salud 2000.Tomo 2. La salud de los adultos. Cuernavaca, México: Instituto Nacional de Salud Pública, 2003. 25. Alvarado-Esquivel C, Sablon E, Martínez-García S, Estrada-Martínez S. Hepatitis virus and HIV infections in inmates of a state correctional facility in Mexico. Epidemiol Infect 2005; I33(4):679-85.

26. Izazola-Licea S, Labastida-Díaz A,Villanueva-Castilleja R. La situación actual de VIH/SIDA en prisiones en México. MEXICO, D.F.: SIDALAC; 1998. 27. Olivero J, Roberts J.AIDS in Mexican prisons.AIDS Soc 1995;6(4):4. 28. Sexual abuse in children and HIV infection (Abs. \#M.D.P. 7).V International Conference on AIDS; 1989; Montreal, Canada. 29.Viloria-Varela G, Montero P. Grupos vulnerables de la Ciudad de México. Entrevista al Diputado por la Asamblea Legislativa del Distrito Federal, Juan Díaz González. Imagen Política de México y el Mundo, 9 de octubre, 2002. 30. Del Río C, Sepulveda J.AIDS in Mexico: lessons learned and implications for developing countries. Aids 2002;16(II): I445-57.

3I.Villasis-Keever A, Rangel-Frausto MS, Ruiz-Palacios G, Ponce de LeonRosales S. Clinical manifestations and survival trends during the first 12 years of the AIDS epidemic in Mexico. Arch Med Res 2001;32(I):62-5. 32. Cámara de Diputados. 59 Legislatura. Centro de Estudios Sociales y de Opinión Pública. Cifras de mortalidad en México, 2003.

33. Secretaría de Salud. Sistema Nacional de Información en Salud. Mortalidad, 2004.

34.Varela-Ramírez A, Mejía A, García D, Bader J,Aguilera RJ. HIV infection and risk behavior of Hispanic farm workers at the west Texas-Mexico border. Ethn Dis 2005; I5(4 Suppl 5):S5: 92-6.

35. Tapia-Aguirre V,Arillo-Santillán E,Allen B,Angeles-Llerenas A, Cruz-Valdez A, Lazcano-Ponce E.Associations among condom use, sexual behavior, and knowledge about HIVIAIDS.A study of 13,293 public school students. Arch Med Res 2004;35(4):334-43.

36. Martínez-Donate AP, Hovell MF, Blumberg EJ, Zellner JA, Sipan CL, Shillington AM, et al. Gender differences in condom-related behaviors and attitudes among Mexican adolescents living on the U.S.-Mexico border. AIDS Educ Prev 2004;16(2): 172-86.

37.Allen B, Cruz-Valdez A, Rivera-Rivera L, Castro R,Arana-García ME, Hernández-Ávila M.Affection, kisses, and condoms: the $A B C$ of sexual practices of female sex workers in Mexico City. Salud Publica Mex 2003;45(Supp 5):S594-607.

38. Pulerwitz J, Izazola-Licea JA, Gortmaker SL. Extrarelational sex among Mexican men and their partners' risk of HIV and other sexually transmitted diseases. Am J Public Health 200 I;91(10):1650-2.

39. Gutiérrez JP, López-Zaragoza JL,Valencia-Mendoza A, Pesqueira E, Poncede-Leon S, Bertozzi SM. [Facing the HIVIAIDS epidemic in Mexico: the response of the health sector]. Rev Invest Clin 2004;56(2):242-52. 40. Izazola-Licea JA, Ávila-Figueroa C,Aran D, Piola S, Perdomo R, Hernández P, et al. Country response to HIVIAIDS: national health accounts on HIVIAIDS in Brazil, Guatemala, Honduras, Mexico and Uruguay.Aids 2002; 16(Suppl 3):S66-75.

salud pública de méxico / vol.49, suplemento 3 de 2007 